\title{
TWO NOTES ON FORMAL POWER SERIES
}

\section{SHREERAM ABHYANKAR}

This paper consists of two more or less disjoint notes, the first on integral formal power series in several variables and the second concerning the generalized Puiseux expansion of a certain algebraic function of one variable over a modular field.

1. Analytically independent formal (integral) power series. Let $k$ be an arbitrary field and let $L_{n}$ be the ring of formal series $k\left[\left[x_{1}\right.\right.$, $\left.\left.x_{2}, \cdots, x_{n}\right]\right]$ in $n$ variables $x_{1}, x_{2}, \cdots, x_{n}$ with coefficients in $k$. We recall that given $m$ elements $f_{i}\left(x_{1}, x_{2}, \cdots, x_{n}\right), i=1,2, \cdots, m$, of $L_{n}$ one says that $f_{1}, f_{2}, \cdots, f_{m}$ are analytically dependent if there exists $0 \neq H\left(Z_{1}, Z_{2}, \cdots, Z_{m}\right) \in k\left[\left[Z_{1}, Z_{2}, \cdots, Z_{m}\right]\right]$ such that $H\left(f_{1}\left(x_{1}, x_{2}, \cdots, x_{n}\right), \cdots, f_{m}\left(x_{1}, x_{2}, \cdots, x_{n}\right)\right)=0$. If $f_{1}, f_{2}, \cdots, f_{m}$ are not analytically dependent then they are said to be analytically independent. Given an infinite number $f_{1}, f_{2}, \ldots$ of elements of $L_{n}$, these elements are said to be analytically independent if every finite number of them are analytically independent. Professor Samuel asked me whether $L_{2}$ contains three analytically independent elements. The answer is given in the following

Proposition. If $n>1$ then $L_{n}$ contains an infinite number of analytically independent elements.

Proof. It is known that there exists an infinite number $g_{1}(y)$, $g_{2}(y), \cdots$ of formal power series in one variable $y$ with coefficients in $k$ which are algebraically independent over $k$ (Lemma 1 of [4]). Let $f_{i}\left(x_{1}, x_{2}, \cdots, x_{n}\right)=x_{2} g_{i}\left(x_{1}\right)$ for $i=1,2, \cdots$. Let $H\left(Z_{1}, Z_{2}, \cdots, Z_{m}\right)$ be an element of $k\left[\left[Z_{1}, Z_{2}, \cdots, Z_{m}\right]\right]$ for which $H\left(f_{1}\left(x_{1}, x_{2}, \cdots, x_{n}\right)\right.$, $\left.f_{2}\left(x_{1}, x_{2}, \cdots, x_{n}\right), \cdots, f_{m}\left(x_{1}, x_{2}, \cdots, x_{n}\right)\right)=0$. Let $H\left(Z_{1}, Z_{2}, \cdots\right.$, $\left.Z_{m}\right)=\sum_{j=0}^{\infty} H_{j}\left(Z_{1}, Z_{2}, \cdots, Z_{m}\right)$ where $H_{j}\left(Z_{1}, Z_{2}, \cdots, Z_{m}\right)$ is a form of degree $j$ in $k\left[Z_{1}, Z_{2}, \cdots, Z_{m}\right]$. Then

$$
\begin{aligned}
0 & =H\left(f_{1}\left(x_{1}, x_{2}, \cdots, x_{n}\right), \cdots, f_{m}\left(x_{1}, x_{2}, \cdots, x_{n}\right)\right) \\
& =\sum_{j=0}^{\infty} x_{2}^{j} H_{j}\left(g_{1}\left(x_{1}\right), g_{2}\left(x_{1}\right), \cdots, g_{m}\left(x_{1}\right)\right) .
\end{aligned}
$$

Therefore

Received by the editors August 23, 1955. 


$$
\begin{aligned}
H_{j}\left(g_{1}\left(x_{1}\right), g_{2}\left(x_{1}\right), \cdots, g_{m}\left(x_{1}\right)\right) & =\text { the coefficient of } x_{2}^{j} \text { in } H\left(f_{1}, \cdots, f_{m}\right) \\
& =0 \text { for } j=1,2, \cdots .
\end{aligned}
$$

Since $g_{1}\left(x_{1}\right), g_{2}\left(x_{1}\right), \cdots, g_{m}\left(x_{1}\right)$ are algebraically independent over $k$, we must have $H_{j}\left(Z_{1}, Z_{2}, \cdots, Z_{m}\right)=0$ for $j=1,2, \cdots$, i.e., $H\left(Z_{1}\right.$, $\left.Z_{2}, \cdots, Z_{m}\right)=0$. Therefore $f_{1}\left(x_{1}, x_{2}, \cdots, x_{n}\right), f_{2}\left(x_{1}, x_{2}, \cdots, x_{n}\right), \cdots$ are analytically independent.

Let us remark that any two elements $f\left(x_{1}\right)$ and $g\left(x_{1}\right)$ of $L_{1}$ are analytically dependent. If either $f$ or $g$ is zero then there is nothing to prove and hence we may suppose that $f\left(x_{1}\right) \neq 0 \neq g\left(x_{1}\right)$. First assume that at least one of the two elements $f\left(x_{1}\right)$ and $g\left(x_{1}\right)$ is a nonunit; say $f\left(x_{1}\right)$ is a non-unit. Then by Proposition 3.5 of Chevalley [2], $L_{1}$ is a finite module over $k\left[\left[f\left(x_{1}\right)\right]\right]$ and hence $g\left(x_{1}\right)$ is integral over $k\left[\left[f\left(x_{1}\right)\right]\right]$. Therefore there exists $0 \neq H(X, Y) \in k[[X]][Y]$ with $H\left(f\left(x_{1}\right), g\left(x_{1}\right)\right)=0$. Now assume that $f\left(x_{1}\right)$ and $g\left(x_{1}\right)$ are both units. Then $f^{*}\left(x_{1}\right)=f\left(x_{1}\right)-a g\left(x_{1}\right)$ is a non-unit, where $a=f(0) / g(0)$. Hence by the previous case, there exists $0 \neq H(X, Y) \in k[[X, Y]]$ with $H\left(f^{*}\left(x_{1}\right), g\left(x_{1}\right)\right)=0$. Let $H^{*}(X, Y)=H(X-a Y, Y)$. Then $H^{*}\left(f\left(x_{1}\right)\right.$, $\left.g\left(x_{1}\right)\right)=0$. Also $H^{*}(X, Y) \neq 0$ since $X \rightarrow X-a Y, Y \rightarrow Y$ is an automorphism of $L_{1}$.

2. A fractional power series. Let $k$ be an algebraically closed field of characteristic $p$. If $p=0$ then the theorem of Puiseux expansion is valid, i.e., any polynomial $F(Y)=y^{n}+f_{1}(X) Y^{n-1}+\cdots+f_{n}(X)$ with $f_{i}(X) \in k(X)$ can be factored in the form

$$
F(Y)=\prod_{i=1}^{n}\left(Y-g_{i}\left(X^{1 / m}\right)\right),
$$

where $m$ is some positive integer and where $g_{1}(X), g_{2}(X), \cdots, g_{n}(X)$ are in the quotient field of $k[[X]]$, i.e., in the integral formal power series field $k((X))$. If $p \neq 0$ then such a factorization is not always possible. A typical example of this is given by Chevalley on p. 64 of [3], namely:

$$
F(Z)=Z^{p}-Z-X^{-1} \text {. }
$$

If we force a factorization, we get the following generalized Puiseux expansion (where the denominators of the indices of $X$ are unbounded):

$$
F(Z)=\prod_{i=0}^{p-1}\left(Z+i-\sum_{i=1}^{\infty} X^{-1 / p^{i}}\right) .
$$

Or getting rid of the poles, we get alternatively: 


$$
Z^{p}-X^{p-1} Z-1=\prod_{i=0}^{p-1}\left(Z+i X-\sum_{i=0}^{\infty} X^{1-p^{-i}}\right)
$$

These factorizations can be verified directly. They were used in discovering some of the examples discussed in [1].

\section{BIBLIOGRAPHY}

1. S. Abhyankar, On the ramification of algebraic functions, Amer. J. Math. vol. 77 (1955) pp. 575-592.

2. C. Chevalley, On the theory of local rings, Ann. of Math. vol. 44 (1943), pp. 680-708.

3. - Introduction to the theory of algebraic functions of one variable, New York, 1951.

4. S. MacLane and O. F. G. Schilling, Zero-dimensional branches of rank one on algebraic varieties, Ann. of Math. vol. 40 (1939) pp. 507-520.

HARVARD UNIVERSITY

\section{ON THE COMPOSITUM OF ALGEBRAICALLY CLOSED SUBFIELDS}

\section{SHREERAM ABHYANKAR}

Professor Igusa asked me the following question: Given a field $K$, is the compositum of all the (absolutely) algebraically closed subfields of $K$ itself algebraically closed (of course, we are assuming that this compositum is not empty, i.e., that $K$ contains the algebraic closure of its prime field)? We shall show in $\$ 1$ that the answer to this question is in the negative in general. In \$2, we shall give a special case in which the answer is in the affirmative.

1. The algebraic closures of $k(x), k(y)$ and $k(x, y)$. Let $k$ be an arbitrary algebraically closed field, $L=k(x, y)$ where $x$ and $y$ are algebraically independent over $k, L^{*}=$ an algebraic closure of $L, M=k(x)$, $N=k(y), M^{*}=$ the algebraic closure of $M$ in $L^{*}, N^{*}=$ the algebraic closure of $N$ in $L^{*}$, and $K=$ the compositum of $L^{*}$ and $M^{*}$. Let $T$ be the compositum of all the algebraically closed subfields of $K$. Then $M^{*} C T$ and $N^{*} \subset T$ and hence $T=K$. We shall prove below that $K$ cannot be algebraically closed. In fact we shall show that in some sense $K$ is much nearer to $L$ than it is to $L^{*}$ and hence that $K$ is far from being algebraically closed.

Embed $L=k(x, y)$ canonically into the formal power series field

Received by the editors August 23, 1955. 